\title{
MATERNIDADE DO EXÍLIO. QUANDO O DESLOCAMENTO FAVORECE A FERTILIDADE
}

Elise Pestre

Elise Pestre

Mestre de conferência na Universidade ParisDiderot, EAD 3522 CRPMS (Centro de Pesquisas em Medicina, Psicanálise e Sociedade), Universidade Paris Diderot, Paris Sorbonne Cité.

Tradução

Fabiana Baptista Mestre em Psicologia pela UFMG e em Psicanálise pela Université Paris 8 Saint-Dennis; doutora em Psicopatologia e Psicanálise pela Université Paris Diderot Sorbonne Cité.
RESUMO: Neste artigo, os efeitos subjetivos da migração em mulheres grávidas em situação de grande precariedade foram explorados a partir de uma pesquisa em psicanálise conduzida no seio de uma maternidade pública da cidade de Buenos Aires (Argentina). A análise dos resultados clínicos mostra que o movimento impulsionado pela migração engendra importantes remanejamentos subjetivos e pulsionais na mulher, dando assim um novo impulso ao encontro com o sexual e à procriação.

Palavras-chave: Migração, gravidez, pulsão, fertilidade.

ABSTRACT: Motherhood in exile. Migration as a stimulus to fertility. Based on a psychoanalytic research project carried out at a maternity hospital in Buenos Aires (Argentina), this paper explores the effects of migration on the subjectivity of pregnant women living in condition of precariousness. The clinical data shows that in these women, the movement initiated by migration provokes a subjective and drive-related reorganisation, which may function as a new stimulus in the encounter with sexuality and procreation. Keywords: Migration, pregnancy, drive, fertility. 
"Aumentarei os sofrimentos da tua gravidez, os teus filhos hão de nascer entre dores."

(Livro do Gênesis)

$\mathrm{N}_{8}$

o livro do Gênesis, Sarai, a mulher de Abraão, é infértil. Seu desejo de engravidar a leva a entregar sua escrava egípcia nos braços de seu esposo, para assegurar a descendência. Será Agar — a estrangeira ou a exilada, em referência à língua árabe ${ }^{1}$ —, que carregará o primeiro filho de Abraão. "Ele possuiu Agar, e ela engravidou”, enuncia o texto bíblico, que revela o imediatismo da gravidez de Agar em terra estrangeira. Ismael nascerá desta união.

Milagrosamente, e contra todas as esperanças, Sarai, que passou a se chamar Sara com a aliança divina, também dará à luz na terra de Canaã. ${ }^{2}$ Com 90 anos, ela colocará no mundo Isaac, cujo significado "Que ele (Deus) ri e sorri, seja favorável"3 advém do caráter estranho dessa gravidez.

Em seguida, temendo as disputas por herança entre os dois filhos de Abraão, Sara tomará a decisão de banir sua escrava e seu filho Ismael. Exilados no deserto e condenados à morte, Agar e Ismael serão, contudo, ouvidos por Deus e salvos do perigo.

Este episódio do Antigo Testamento permite entrever como, desde a Gênese, o exílio, a fertilidade e a maternidade estão ligados de maneira irredutível. Esta intrincação originária aponta o deslocamento e a fertilidade como sendo acontecimentos universais, desde a fundação do homem monoteísta.

A partir de uma pesquisa realizada na Argentina, em uma maternidade pública da cidade de Buenos Aires, veremos como o deslocamento é revestido de “destinos particulares” (FREUD, 1915) e favorece amarrações pulsionais inéditas, mas às vezes perigosas para o sujeito. À imagem das personagens bíblicas Sara e Agar, a pesquisa denominada "Os efeitos subjetivos do deslocamento em mulheres migrantes”4 mostra a maneira como o exílio é suscetível de produzir consequências singulares na capacidade da mulher de procriar em terra estrangeira. Consideraremos, assim, os cruzamentos e os “descruzamentos” entre os territórios do corpo biológico e do corpo pulsional.

\footnotetext{
1 "O nome (Hajar) é ligado ao de Hagrites, tribos nômades. O nome Hajar tem como raiz h.j.r. A raiz etimológica significa exílio”, in $\mathrm{H}$. Abdelahoued, Figures du féminin en Islam. Paris : PUF, 2012, p.61. (Petite Bibliothèque de Psychanalyse)

${ }^{2}$ Os três protagonistas vêm de outro território: Abraão e Sarai são originários de Ur, na Caldeia e Agar é egípcia.

${ }^{3}$ Sara ri, quando, apesar de sua idade, lhe é anunciada que sua gravidez se aproxima (Gênesis $16: 2$ e 26:6).

${ }^{4}$ Esta pesquisa foi realizada em 2009, em Buenos Aires (Argentina) e foi conduzida graças à colaboração da equipe do serviço de saúde mental do Hospital Ramón Sardá e da Universidade de Psicologia de Buenos Aires (UBA — Mestrado em Políticas de Migraciones Internacionales).
} 
Veremos que o movimento subjetivo impulsionado pelo deslocamento territorial parece possuir o poder de desamarrar certas junções pulsionais até então em ação. Entretanto, seria realmente o atravessamento das fronteiras territoriais que influenciaria no desejo de engravidar ou na fertilidade da mulher? Qual é o lugar ocupado pelo sexual infantil na ativação destas gestações em terra estrangeira? Em que ponto a gravidez na terra do exílio se diferencia da gravidez na terra natal? Tentaremos trazer aqui alguns elementos de resposta a estes questionamentos.

\section{UMA CLÍNICA NA CABECEIRA DAS PACIENTES}

$\mathrm{Na}$ maternidade Sardá, ${ }^{5}$ os funcionários circulam entre as camas, em meio a grandes dormitórios coletivos onde as mulheres grávidas estão acamadas por causa de uma gravidez patológica. Durante as entrevistas, os psicólogos clínicos do serviço de saúde mental assentam-se ao lado das pacientes — ou mesmo na cama delas ou em uma cadeira ao lado — dando a ver o exercício de uma verdadeira Kline. ${ }^{6}$

Foram entrevistadas 40 pacientes no decorrer desta pesquisa qualitativa, que se situa na interseção entre a psicanálise, a medicina e a clínica transcultural.

A metade das mulheres entrevistadas estava hospitalizada no local, em função de uma gravidez de risco (hipertensão, diabetes, ruptura prematura da bolsa das águas, etc.); a outra metade vinha em consulta externa e aguardava na sala de espera (para urgência ou simples controle). Eu encontrei as pacientes somente uma vez, pois realizei apenas uma entrevista com cada uma delas. ${ }^{7}$ As entrevistas foram realizadas em espanhol, língua materna ou adotiva das mulheres entrevistadas, sendo que algumas delas falavam dialetos regionais (o quéchua e o guarani, principalmente).

Estas mulheres grávidas não apresentavam nenhum transtorno psicopatológico anterior à gravidez (somente uma delas evocará um tratamento psicoterapêutico). Em essência, originárias de países limítrofes da Argentina (Bolívia 24\%, Paraguai 11,6\% e Peru 5,5\%), menos de um quarto delas eram primíparas. As pacientes entrevistadas - selecionadas graças à orientação das equipes ou de maneira

\footnotetext{
${ }^{5}$ Situada no sul da capital, Sardá é a maternidade mais importante da Argentina. Credenciada pela Unicef e pelo Ministério da Saúde, este estabelecimento realiza aproximadamente sete mil partos por ano. Informações no site http://www.sarda.org.ar/.

${ }^{6}$ Lembramos aqui que esta palavra grega significa "leito, cama", derivada do verbo grego klinein, "inclinar".

${ }^{7} \mathrm{O}$ projeto inicial desta pesquisa tinha por objetivo promover vários encontros com as pacientes migrantes, ao longo da gravidez. Entretanto, foi extremamente difícil realizar mais de uma entrevista com as mesmas pacientes, em função da precariedade que impedia as mulheres de antecipar os momentos das entrevistas. Em termos metodológicos, tivemos que nos adaptar a esta realidade e, assim, fazer a escolha de realizar uma única entrevista.
} 
espontânea nas salas de espera - estavam presentes em solo argentino entre um e dez anos, no máximo. A idade média era de pouco menos de 25 anos. ${ }^{8}$

Nesta maternidade pública, as mulheres se consultam gratuitamente. Muitas moram na periferia da cidade e a maioria vive em uma precariedade importante, em lugares insalubres ou mesmo em favelas.

Mais de 40\% das mulheres que se consultam são migrantes. Quando o deslocamento é motivado por razões econômicas e sociais, as mulheres cruzam a fronteira sozinhas ou com o companheiro. Quando a migração é realizada antes da maioridade, elas em geral se mudam com os pais. Com frequência, os filhos de uma primeira união continuam no território de origem, com parentes. Os casais que se formam na terra de adoção são, em 90\%, compostos por um membro vindo da mesma minoria.

\section{NOVOS TERRITÓRIOS "FERTILIZANTES"}

A questão da possibilidade de procriar em terra estrangeira se mostrou, rapidamente, como um dos pontos centrais da nossa pesquisa. Com efeito, a análise do discurso das mulheres entrevistadas dá conta de uma vivência e de uma realidade contrastadas quanto à capacidade delas em procriar na terra de adoção.

Um dado chamou nossa atenção: mais de três quartos das 40 mulheres entrevistadas começaram uma gestação ao longo dos dois primeiros anos de migração. ${ }^{9}$

Esta espécie de prontidão para engravidar nos interpela como clínicos, em especial porque a maioria dessas mulheres não estava necessariamente inscrita em um programa de "gravidez voluntária" no momento em que elas ficaram grávidas. ${ }^{10}$ Este dado, consequência da gravidez que surge no momento de uma “onda migratória”, nos questiona. É como se o fato de ultrapassar as linhas sim-

\footnotetext{
${ }^{8}$ As entrevistas semiestruturadas foram realizadas a partir de diversos eixos: elementos de ordem sociológica - emprego, lugar de moradia; motivos da migração e vivência subjetiva da mulher em relação a este deslocamento; temporalidades em jogo no fato de ficar grávida; vivência desta gestação fora do país de origem, dores relativas à gravidez, etc.

${ }^{9}$ Entre estas mulheres, a metade começou uma gravidez logo no primeiro ano de presença no solo argentino.

${ }^{10}$ Em espanhol, a palavra recorrentemente utilizada para falar de uma gravidez é "caer" grávida, "caer embarazada”, o que daria em português, literalmente "ficar grávida". Existe aqui um jogo de palavras interessante, referente à riqueza do significante caer, comum entre as línguas espanhola e francesa e que forma a expressão em espanhol caer embarazada, e em francês tomber enceinte. Se o verbo "tomber" em francês indica a ideia de queda em uma dimensão repentina, "ser levado para baixo sob o peso da gravidade", ele envia também a uma multitude de significações. Mais particularmente, o verbo indica "privar de reputação, de crédito". Ou ainda, como mencionado na oitava edição do dicionário da Academia francesa, "o fato de se tornar insensível às verdades da religião. Cair no pecado, cometer uma falta, ceder ao pecado”. Sabendo que várias mulheres entrevistadas são católicas, estas referências religiosas adquirem um interesse particular no contexto do nosso estudo.
} 
bólicas e imaginárias das fronteiras influenciasse as coordenadas biológicas e corporais da mulher, favorecendo e acelerando, inconscientemente, o despertar da maternidade e da gestação.

Embora muitas vezes estas mulheres não desejem procriar, elas se encontram, no entanto, embaraçadas ${ }^{11}$ com essa gestação, pouco depois da chegada delas na terra de adoção. Como o aborto na Argentina é proibido, a gravidez em geral é levada até o fim, a não ser que aconteça algum acidente ou aborto ilegal. Para além dos erros de contracepção - notemos que esta última é pouco utilizada pelas mulheres entrevistadas e que muitas delas praticam a contracepção "natural" —, a capacidade de procriar toma novo fôlego, logo que o deslocamento é realizado.

Mencionemos ainda que, desde os anos 1970, o essencial da literatura sobre a relação entre migração e fertilidade pertence aos campos da medicina e das ciências sociais. O demógrafo inglês Hill Kulu mostrou, por exemplo, que as taxas de fecundidade são maiores depois de realizadas mudanças do local de residência (KULU, 2005, p.51-87). Entretanto, este autor demonstra, com base em uma importante revista da literatura anglo-saxônica contemporânea, que os estudos sobre este tema são divergentes. Ressaltamos também que eles se inscrevem, em sua maioria, em estudos demográficos e étnicos muitas vezes marcados por uma dimensão neomalthusiana da fecundidade, que preconiza restrições demográficas. Por fim, são os trabalhos de migrations studies (HERVITZ, 1985, p.293-317) e gender studies (ESPIN, 1999) sobre a temática "migração e sexualidade" que se revelam ser, no nosso ponto de vista, os mais pertinentes. Esta é a razão pela qual queremos abordar este estudo a partir da perspectiva psicanalítica, para identificar as relações singulares existentes entre migração, fertilidade e subjetividade.

O deslocamento territorial parece operar como um "fertilizador" nas mulheres em estado migratório. Esta constatação levanta várias questões, ainda mais quando se considera que as mulheres que não conseguem engravidar no país de origem conseguem ficar grávidas na terra de adoção. De fato, várias mulheres consideradas até então como estéreis antes da migração ficaram grávidas pouco depois da chegada ao novo território, sem que nenhuma intervenção médica tenha sido realizada. Assim que a infertilidade e a esterilidade desaparecem e se resolvem espontaneamente, esta gravidez ganha grande interesse científico.

\footnotetext{
${ }^{11}$ O termo utilizado aqui é, em francês, "embarrassées" (embaraçada), que guarda uma relação direta com a palavra "embarazada", que em espanhol significa "grávida". A tradução da palavra francesa "embarrasée" era utilizada na França antigamente, e se referia ao fato de uma mulher ficar grávida sem ser casada. Além disso, este termo evoca também a dimensão passiva e problemática deste estado transitório e o embaraço que ele suscita nos trabalhos domésticos.
} 
O caso de uma dessas mulheres, Mariana, ajudará na compreensão das questões suscitadas por este enigma biológico.

\section{MARIANA OU A FERTILIDADE INEXPLICADA ${ }^{12}$}

Quando encontro Mariana na sala de espera da maternidade para uma consulta de controle, ela me diz ainda estar "em estado de choque" com o anúncio de sua gravidez. Ela tem 27 anos e foi diagnosticada estéril em seu país de origem, o Paraguai, por causa de uma malformação uterina, segundo ela. ${ }^{13}$ Ela me diz que "ainda não consegue acreditar nisso". Quando eu a questiono sobre o que ela sentiu com a notícia, ela pontua: “eu não esperava por isso. Lá (no Paraguai), eles me disseram que eu não poderia ter filhos e que eu não tinha o útero desenvolvido o bastante". Mariana divide sua vida com o mesmo companheiro desde que começou a tentar engravidar há três anos e meio em seu país de origem. Há apenas um ano, o casal se instalou em Buenos Aires para "mudar de vida" e “encontrar um trabalho”. Ela só percebeu que estava grávida com quatro meses de gestação. Quando eu a encontro pela primeira vez, ela está grávida de mais de cinco meses e sua barriga é bem pequena.

Ao longo da entrevista, a paciente diz ter tido muitos pesadelos em relação à gravidez: "eu sonho com coisas feias, com perdas. Acordo à noite por causa disso", diz ela. O significante "perda” que Mariana utiliza tem uma forte ressonância. É difícil nos impedir de pensar em sua família que mora longe. Suas condições de vida precárias a impedem de viajar para visitar seus parentes. Ela acrescenta, pouco depois: "sinto muitas saudades da minha mãe".

A recorrência dos sonhos de perda de Mariana, essas “coisas feias” que a acordam à noite, parece testemunhar uma angústia em função da distância criada por esta oscilação inesperado entre o desejo de ter um filho e a concretização da gravidez. De maneira geral, no momento da gestação, o inconsciente mostra que o desejo de engravidar nunca é "um desejo puro" (BYDLOWSKI, 2010, p.213, totalmente livre de uma carga ambivalente. Os desejos de morte estão sempre presentes, como filigranas, independente se o desejo de ter uma criança tenha precedido ou não a gravidez. Mas, para Mariana, esta novidade é recoberta por um caráter vertiginoso: o anúncio da gravidez, que é sempre o de “dar a

\footnotetext{
${ }^{12}$ Retomo, aqui, por inversão, a expressão médica atualmente utilizada, a saber infertilidade inexplicada. Esta expressão nos reenvia à impossibilidade de concepção, depois de dois anos de relações sexuais regulares, mesmo que todas as explorações médicas complementares da pesquisa diagnóstica tenham sido consideradas satisfatórias.

13 A paciente foi precisa: "eu tinha um útero muito pequeno". De acordo com o dossiê médico, diversas análises médicas concluíram por uma hipoplasia uterina bastante grave. Entretanto, as análises não estavam anexadas ao dossiê médico. Assim, dificilmente podemos trazer mais elementos sobre isso.
} 
morte” (BENSLAMA, 2005, p.68), libera um conteúdo arcaico recalcado. De acordo com a concepção de Fethi Benslama, “independentemente do conteúdo do anúncio - a vinda de uma criança, o texto, o sacrifício, o novo tempo, etc. - ele aparecerá sempre como sendo a revelação de uma responsabilidade diante do outro que ultrapassa o que é possível, pois ele encarna um "dar a morte" exatamente pelo fato de dar a vida”. De fato, carregar a vida é também carregar a morte. O trabalho do sonho se desdobra aqui a fim de integrar e simbolizar o anúncio inesperado da gravidez. O futuro bebê veste, fantasmaticamente, as vestimentas da morte e as representações de um aborto espontâneo, arruinando este futuro acontecimento feliz.

Se a infertilidade pode se decifrada tal como um sintoma, que revela certa disjunção do sujeito, seu desaparecimento repentino, sem intervenção de nenhuma técnica médica, é para nós testemunha da manifestação de uma verdade subjetiva. Ele dá a ver toda a dimensão psíquica da infertilidade. ${ }^{14}$ Com efeito, o fato de que esse sintoma desapareça logo depois do deslocamento territorial, questiona as modificações inconscientes ligadas à capacidade biológica de poder engravidar. Inclusive, Mariana, ainda siderada por este anúncio, encontra como única resposta a este enigma biológico um presságio divino. A ausência de explicação científica para sua gravidez aponta para o caráter milagroso desta gestação. O desenrolar da gravidez é colocado nas mãos de Deus, que interviu, em terra estrangeira, sobre uma mulher que não acreditava mais em sua capacidade de engravidar. De fato, quando a questiono sobre sua gravidez, Mariana responde: "é porque Deus quer".

A queda misteriosa do sintoma de infertilidade mostra a força extraordinária que o inconsciente exerce sobre o corpo: o diagnóstico médico de esterilidade é varrido em benefício de uma fertilidade medicamente inexplicada. Transportados para outra realidade - soma e psiquê se reconfiguram de maneira diferente. O surgimento deste novo estado dá a ver os efeitos de tradução psíquica e somática que acontecem em função do deslocamento de território, favorecendo assim os processos de simbolização.

A infertilidade, solidamente mantida pelo recalque, é então destituída para realizar aqui uma nova ligação que se realiza nos investimentos libidinais e em uma ativação espontânea da gravidez.

\footnotetext{
${ }^{14}$ Desde os anos 1970, importantes estudos clínicos propuseram relações entre infertilidade e psiquismo a partir de diferentes paradigmas. O autor Greil propõe uma revista: GREIL, AL (1977), "Infertility and psychological distress: a critical review of de literature", Soc Sci Med 1977; 45:1689-1704. Entre os trabalhos atuais, ver: FAURE-PRAGIER, S. (1993), BYDLOWSKI M. (1997), STOLERU et al: "Psychological characteristics of infertile patients:discriminating etiological factors from reactive changes”, J. Psychosom Obstet Gynaecol 1996; 17:103-118.
} 
Para Mariana, como para outras mulheres, a mudança de país terá sido necessária para que a gravidez pudesse ser declarada. Exploraremos agora as hipóteses clínicas que permitem pensar em uma articulação possível entre deslocamento e fertilidade.

\section{ENCONTRO COM O SEXUAL}

A transposição das fronteiras esconde um efeito de emancipação que faz do deslocamento um acontecimento propício ao encontro com o sexual e à realização de desejos ligados a ele. O corpo pulsional, de início regulado pela cultura de origem, encontra-se repentinamente “desbloqueado". Em termos edipianos, a cultura do pai, cujos referentes tradicionais presentes no seio da população entrevistada estão impregnados pelo catolicismo, com todas as interdições da sexualidade que esta religião implica, parece ser relegado para segundo plano.

O distanciamento da terra de origem parece produzir uma queda da autoridade do supereu que favorece a reaproximação com a pulsão. A libido pode agora circular e se escoar sem fronteiras, longe dos olhares reprovadores ligados à terra dos antepassados. Inclusive, esse “desbloqueio" da sexualidade, tanto simbólico quanto real, se exprime com lucidez em mulheres mais jovens, que formulam de uma maneira ou de outra que "isso não teria acontecido” no seu país de origem. Sandra, uma jovem adolescente boliviana de 17 anos e grávida de oito meses, dirá: “com certeza, eu não teria ficado grávida lá (na Bolívia). É outra cultura, a gente não sai tanto. Aqui as meninas são loucas e isso é contagiante!”. Nesse sentido, o exílio, com o deslocamento que ele implica, representa uma queda do olhar paterno, que é o mesmo olhar que modera o gozo.

É como se, pouco depois da mudança territorial, a pulsão, que caminha em direção à satisfação, se lançasse rapidamente para uma descarga, e em seguida, se apoiasse com força para voltar rapidamente para sua fonte. Esta última se localiza em um enraizamento somático, lugar onde, precisamente, a fertilidade se materializa. Eros parece aqui desdobrar suas pulsões de vida ao estado puro pelo viés desta "incorporação de um desejo inconsciente” (ASSOUN, 1992, p.59). Ele trabalha assim para o destino da pulsão, quando a "necessidade inexorável - e as vicissitudes contingentes” (ASSOUN, 1997, p.396) relativas à procriação, se instauram. Esta gestação parece ter como último desígnio dominar a formidável força das pulsões sexuais pelo intermédio deste corpo grávido, que está, de agora em diante, bloqueado de maneira diferente. Agora, a gestação obstrui a pulsão e parece vir no lugar de um sexual inconfessável ou 
inconciliável. O "inchaço na barriga"15 é a marca do retorno do recalcado que aparece no corpo da mulher.

A migração, seguida da gestação, dá a ver esta gravidez como tendo uma significação essencialmente nostálgica. O distanciamento do país natal engendra a dimensão do nostalgos, esta tristeza ligada ao pesar por ter deixado a terra de origem. Neste novo contexto, a criança no ventre se materializa como "prova de amor, como um presente" (FREUD, 1917/1997) oferecido ao pai distante, à sua pátria. ${ }^{16}$ Inclusive, nesta perspectiva, o parceiro sexual representaria um substituto fantasmático do pai, como uma espécie de prolongamento fantasmático edipiano (BRUN \& MOURAS, 2003). A doação ao pai se concretizaria neste espelhamento com o parceiro, justamente para poder se reaproximar deste pai de quem ela se distanciou geograficamente.

Mas as tentativas de reencontros com a terra natal e com os próximos são dúbias. Para além do pai, trata-se também, para a mulher grávida, de reencontrar a mãe dos tempos pré-edipianos, ${ }^{17}$ pois se a terra é pater ela é, antes de tudo, mater, terra mãe.

\section{A (PRO)CRIAÇÃO DE UM ALTER EGO}

A busca de uma nova morada passaria, para algumas mulheres migrantes, pela materialidade de um corpo em gestação? Quando a experiência do sujeito em busca de hospitalidade foi difícil, ela se traduziria pela fabricação de um alter ego, que se instala no corpo próprio da mulher que é em situação de migração. A criação, no interior de si, de um outro semelhante teria, fantasmaticamente, vertentes protetoras e de contenção pelo xenos, o estrangeiro. De fato, aquela que se sente isolada e angustiada poderia assim se refugiar imaginariamente neste lugar mesmo em que ela (pro)criou, a fim de tentar atenuar isso.

No momento do encontro do sujeito com a sua própria alteridade na nova terra, os afetos liberados podem se revelar fortes, e às vezes desestabilizastes e destrutivos. No momento da gravidez, esta vivência será, por vezes, amplificada e materializada no corpo, ainda que o desejo primeiro fosse de sufocá-los, de remediá-los por meio desta gestação. O estado depressivo, encontrado com

\footnotetext{
${ }^{15}$ A expressão, no original, é “bosse au ventre”. Trata-se de uma antiga expressão francesa que pertence ao registro familiar. Ela faz referência ao inchaço devido à uma contusão óssea. Pode-se referir também, de maneira pejorativa, à mulher que recebeu um "golpe" na barriga e ficou inchada, ou seja, grávida. O interessante dessa expressão é mostrar que esse "choque, golpe" recebido deixa uma marca ("bosse") no soma.

${ }^{16}$ A palavra pátria é derivado de pater, pai e significa "país onde se nasce”.

${ }^{17}$ Monique Bydlowski mostrou a dupla vertente edipiana e pré-edipiana do desejo da criança e do processo de maternidade em seus textos, principalmente em Bydlowsky M., La dette de vie, Itinéraire psychanalytique de la maternité, PUF, Le fil rouge, Paris, 1997.
} 
frequência nas mulheres migrantes grávidas, já foi levantado em alguns estudos clínicos. ${ }^{18}$ Os resultados mostram que a gravidez "em outro lugar" pode se constituir como um tempo em que a tristeza e o sentimento de solidão fluem de maneira particular. Se um dos objetivos inconscientes desta gestação é de reduzir a angústia e o isolamento, a gestação não resolve necessariamente esta equação nostálgica, pois o sentimento de "continuidade da existência” (WINNICOTT, 1969) dos primeiros estágios da vida fica, cruamente, colocado à prova.

Entretanto, em alguns casos, a maternidade se configura como um espaço intermediário que oferece a possibilidade de um entre dois lugares. O bebê encontrado-produzido entra em relação direta com a parte inconsciente do Eu da futura parturiente. Aqui, a adaptação das defesas, tanto corporais quanto psíquicas, permite lutar contra o estranhamento provocado devido ao distanciamento e à angústia que ele quase sempre suscita. Nesta perspectiva, um autor como J. Raphael-Leff pode evocar a função de "contenção da procriação" (procreative containment), em que o ventre materno aparece como um espaço transformador dos elementos beta, tóxicos, em elementos alpha, não tóxicos (BION, 1962). ${ }^{19}$

Assim, se produzira na mulher uma forma de injunção inconsciente do psiquismo para que se forme um alter ego, colocando a gestação em terra estrangeira do lado do narcisismo primário e das pulsões de autoconservação. Assim, a busca de uma morada psíquica e territorial passaria por esta etapa.

É pelo viés desta necessidade de engravidar que a mãe dos tempos préedipianos é convocada. Inclusive, e menos metaforicamente, a gestação da mulher oferece a possibilidade real do deslocamento da sua genitora: na maioria dos casos, a mãe visita a filha no momento da gravidez ou na época do nascimento da criança, independente de suas condições socioeconômicas. Em suma, é como se, de repente, a mãe rival do Édipo, que foi a princípio colocada de lado - desalojada com o exílio? - pudesse ser aqui reencontrada. O processo de gestação oferece a possibilidade de uma nova ligação materna, favorecendo assim uma aproximação com a (terra) mãe.

\section{UMA DOLOROSA GESTAÇÃO NA TERRA DO EXÍLIO}

Por outro lado, a análise do discurso das mulheres entrevistadas ressalta que para a maior parte delas — seja a gestação normal ou patológica — a gravidez na terra do exílio se revela "dolorosa”. Este dado sobre a vivência somática da gestação

\footnotetext{
${ }^{18}$ Ver principalmente os trabalhos de Zelkowitz P., Schinazi J., Katofsky L., Saucier, J.-F. et al. (2004), "Factors associated with depression in pregnant immigrant women", Transcultural Psychiatry, 41, p.445-464.

${ }^{19} \mathrm{O}$ autor fala de "imunidade psicológica", mas nós preferimos falar aqui em termos de imunidade psíquica.
} 
das mulheres migrantes é ainda mais evidente quando outra gravidez aconteceu no país de origem, antes da migração. Neste caso, a gestação passada é sempre comparada à atual, e distinta desta pela negativa. Os diversos comentários ilustram isso: "este aqui mexe mais", ou ainda "eu não sei se eu sinto mais dor, mas todo o meu corpo me faz mal, a barriga está maior, ela está diferente da primeira vez e mais pesada também”; “a primeira não me fazia mal. Esta é mais difícil”.

O discurso contrastado entre as gravidezes vividas lá e aqui nos leva a recontextualizar a vivência de um corpo que aparece afetado por esta gestação exilada, como se a mudança de território viesse a se encarnar na mulher. Em termos cirúrgicos, por exemplo, diz-se que encarnar significa a "produção de carne em reparação a um corte". ${ }^{20}$ Em alguns contextos psíquicos depressivos, o feto não apareceria como uma produção de carne viva, que tenta reconstituir o corte psíquico relativo ao distanciamento e às separações? Em muitas mulheres migrantes entrevistadas, os afetos depressivos se manifestam pelo viés de um corpo em sofrimento. ${ }^{21}$

Enfim, e como há um preço a pagar por essa emancipação familiar, o bebê "que faz mal" não apareceria, em alguns casos, como uma sentença punitiva diante da culpabilidade revelada pela sexualidade em jogo? Esta sentença estaria ligada à culpabilidade realativa ao pecado original consumado: o de ter comido o fruto proibido.

\section{CONCLUSÃO}

A partir desta pesquisa em perinatalidade, podemos observar um antes e um depois relativo ao deslocamento e à procriação: verdadeiros efeitos de tradução são produzidos na economia inconsciente da mulher. O que estava entravado, ou mesmo intraduzível (ALTOUNIAN, 2005) se move no momento desta nova instalação territorial e consegue se materializar de outra maneira, pelo viés do corpo "grávido" da futura parturiente. Com a migração, importantes modificações subjetivas e somáticas podem então acontecer.

Os desejos de emancipação familiar se intrincam diretamente aos desejos de reencontros edipianos e pré-edipianos. Tais votos inconscientes se revelam diretamente ligados à nostalgia do pai, e à identificação primária ao "pai da pré-história pessoal” (FREUD, 2010), identificação esta que está presente no

\footnotetext{
${ }^{20}$ Segundo o dicionário francês Emile Littré, o termo encarnação se refere, em psicologia, à "produção de um germe ou embrião no óvulo". A palavra vem do latim incarnatio, que significa a "ação de tomar corpo", Littré, Encyloepedia Britannica France Ltd, t.3, Versailles, 1993, p.3.149.

${ }^{21}$ Estas manifestações corporais nas populações migrantes e refugiadas foram largamente desenvolvidas, consultar principalmente: Douville, O., Galap, J., “Santé mentale des migrants et réfugiés en France”, Encyclopédie Médico-chirurgicale, Psychiatrie, Elsevier, Paris, 1999, p.37880-A-10 e Pestre E., La vie psychique des refugies. Paris: Payot, 2010.
} 
fundamento da religião. Por fim, e fantasmaticamente, seria possível, na terra do exílio, dar à luz, ser ouvido e salvo, como Agar e Ismael, que errantes no deserto foram salvos pela força do pai omnisciente e indulgente, apesar das tentativas de emancipação da terra de origem.

Assim, parece que, para algumas mulheres migrantes, a gestação oferece a esperança de um refúgio, ainda mais quando a saída do país de origem e o deslocamento representam para elas eventos marcantes. A gravidez e o nascimento da criança na terra de acolhimento poderão se constituir num vetor do laço social favorecendo assim a produção de um habitat para a mãe e o casal, desde que ela esteja em condições de se apropriar subjetivamente de sua gravidez. Esta apropriação será facilitada — ou mesmo possibilitada — graças à hospitalidade e ao acompanhamento clínico oferecido pelo país hospedeiro, que sozinho oferecerá a possibilidade de uma real inscrição política, psíquica e social da parturiente.

Recebido em 26/7/2013. Aprovado em 20/8/2013.

\section{REFERÊNCIAS}

ABDELAHOUED, H. (2012) Figures du féminin en Islam. Paris: PUF. (Petite Bibliothèque de Psychanalyse)

ALTOUNIAN J. (2005) L'intraduisible. Deuil, mémoire, transmission. Paris: Dunod. (Coll. Psychismes)

ASSOUN, P.-L. (1992) “Le désir machinal de l'enfant”, Les cahiers de l'IPPC, Le petit de l'homme, l'enfant de la machine, Institut de psychopatologie clinique, n 13, avril, p.55-78.

ASSOUN, P.-L. (1997) Psychanalyse. Paris: PUF.

BENSLAMA F. (2005) “De la responsabilité de l'annonce”, Violence de l'annonce, violence du dire, VII Colloque de Médecine et psychanalyse (sous la direction de Brun), Ed. Etudes freudiennes, janvier, p.67-72. BOUKOBZA, Claude (2003) "La clinique du holding Illustration de D.W. Winnicott”, Le Coq-héron, 2 n.173, p. 64-71.

BRUN C., MOURAS J.-P. et al. (2003) La périnatalité : repères théoriques et cliniques 1 et 2 cycle. Amphi Psychologie, Bréal, Rosny-sous-Bois.

BYDLOWSKI, M. (2010) "Que dire du désir d'enfant ?", Le statut de la femme en médecine, entre corps et psyché, XIème Colloque de Médecine et psychanalyse, Études freudiennes hors-série, p.211-216.

(1997) La dette de vie, Itinéraire psychanalytique de la maternité. Paris: PUF (Le fil rouge)

DOUVILLE, O., GALAP, J. (1999) “Santé mentale des migrants et réfugiés en France”, Encyclopédie Médico-chirurgicale, Psychiatrie, Elsevier, Paris, p.37-880-A-10. 
ESPIN, O. M. (1999) Women Crossing Boundaries: The Psicology of Immigration and the Transformations of Sexuality. New York: Routlege.

FAURE-PRAGIER, S. (1997) Les bébés de l'inconscient. Le psychanalyste face aux stérilités féminines aujourd'hui. Paris : PUF.

FREUD, S. (2010) Le Moi et ça. Paris: Payot. (Petite Bibliothèque) . (1917/1997) "Sur les transpositions de pulsions plus particulièrement dans l'érotisme”. La vie sexuelle. Paris: PUF. (1915) Métapsychologie, GW, X.

GREIL, A. (1977), Infertility and psychological distress: a critical review of the litterature, Soc Sci Med; 45:1679-1704.

HERVITZ, Hugo M. (1985) “Selectivy, Adaptation, or Disruption? A Comparison of Alternative Hypotheses on the Effects of Migration on Fertility: The Case of Brazil, International Migration Review, v.19, n.2 (summer), p.293-317.

KULU, H. (2005) "Migration and fertility: competing hypotheses reexamined”, European Journal of Population, 21: 51-87.

PESTRE E. (2010) La vie psychique des réfugiés. Paris: Payot.

RAPHAEL-LEFF, J. (1996), Pregnancy: Pregnancy- Procreative Process, The Placental Paradigm and Perinatal Therapy, J. Amer. Psychoanal. Assn., 44S:373-39.

REY, A. (sous la dir. de) Le grand Robert de la langue française, Dictionnaire Le Robert, Paris, 2001.

STOLERU et al. (1996) Psychological characteristics of infertile patients: discriminating etiological factors from reactive changes, J. Psychosom Obstet Gynaecol, 17:103-118.

WINNICOTT, D.-W. (1969), “La capacité d’être seul”, De la pédiatrie à la psychanalyse. Paris: Payot.

ZELKOWITZ P., SCHINAZI J., KATOFSKY, L., SAUCIER, J.-F et al. (2004) Factors associated with depression in pregnant immigrant women, Transcultural Psychiatry, 41, p.445-464. Dictionnaire TLFI, Cnrtl, CRNRS Edition, http://www.cnrtl.fr

Elise Pestre

elise.pestre@gmail.com

Fabiana Baptista

fabibap77@hotmail.com 